\begin{tabular}{cc} 
Sharif University of Technology \\
Scientia Iranica \\
SCIENTIA & Transactions F: Nanotechnology \\
I RAN I CA & \\
\hline
\end{tabular}

\title{
The effects of laser wavelength and particle size on heating and melting of gold nanoparticles dispersed in liquid
}

\author{
S. Malek ${ }^{\mathrm{a}}$ and R. Poursalehi ${ }^{\mathrm{b}, *}$ \\ a. Department of Physics, Faculty of Basic Sciences, Shahed University, Tehran, Iran. \\ b. Nanomaterials group, Department of Materials Engineering, Tarbiat Modares University, Tehran, P.O. Box 14115-143, Iran. \\ Received 27 January 2014; received in revised form 6 September 2015; accepted 25 April 2016
}

\author{
KEYWORDS \\ Nanoparticle; \\ Optical absorption; \\ Laser wavelength; \\ Melting; \\ Photothermal heating.
}

\begin{abstract}
In this research, the heating process and melting of water surrounded homogenous gold nanospheres irradiated by nanosecond laser pulses at the wavelengths of $355,532,633$, and $900 \mathrm{~nm}$ are studied. The estimation of absorbed energy by gold nanoparticles with radius range 1-40 $\mathrm{nm}$ and their maximum temperature is done using the absorption efficiency of the nanoparticles at the corresponding laser wavelength. The dependency of the melting temperature of nanoparticles upon their size is also considered. It is seen that progress in the melting process strongly depends on the laser wavelength and particle size. The laser wavelength of $532 \mathrm{~nm}$ has been found appropriate for effective photothermal heating of a large gold nanoparticle. Controlling the laser irradiation wavelength is crucial to achieve the best conditions for the desired applications such as localized heating and nanowelding.
\end{abstract}

(C) 2016 Sharif University of Technology. All rights reserved.

\section{Introduction}

Employing pulsed lasers in nanofabrication processes has been a source of great interest as reported by Kadhim et al. [1]. Pulsed laser interaction with metal nanoparticles dispersed in liquid is a fundamentally intriguing issue and is also a basis for a number of emerging applications including laser-assisted size reduction, formation of networked nanoparticles, alloy nanoparticles formation, and nano-joining as studied by Mafune et al. [2]. Muniz-Mirande et al. [3] employed pulsed laser heating, melting, and ablation as alternative approaches for efficient material nano-structuring. In addition, size reduction by fragmentation of gold

\footnotetext{
*. Corresponding author. Tel.: +98 2182883997 ; Fax: +982182884390

E-mail addresses: poursalehi@tmu.ac.ir (R. Poursalehi); s.malek@shahed.ac.ir (S. Malek)
}

nanoparticles under laser exposure has also been reported by Warner et al. [4].

Laser illumination and heating has been progressively used to adjoin, to hold closely, and to weld nanoparticles as a powerful tool for joining and surface engineering of nanomaterials as investigated by Son et al. [5]. For instance, gold@silver core@shell composite nanowires could be obtained by silver nanojoining of gold nanoparticles under optimum condition of laser energy density of $532 \mathrm{~nm}$ laser pulse [5]. Differences in optical properties of base-metallic and filler-metallic, especially difference in size-dependent surface plasmon properties, play an important role in appropriate nanojoining [5]. Laser-induced nanomaterials processing can be achieved by considering the size-dependent thermal properties of metal nanoparticles. In this regard, Mafune et al. [6] reported pulsed laser soldering of platinum and gold nanoparticles dispersed in water. In addition, nanoparticles would be greatly beneficial 
in welding for soft materials and nano-devices due to the less value of temperature as investigated by Cui et al. [7] and Guo et al. [8].

Photothermal processes based on laser nanomaterials interaction such as laser nanojoining, nanowelding, and nano-soldering have created opportunities to manufacture various nano-devices as detailed by $\mathrm{Hu}$ et al. [9], Kim and Jang [10], Zhou et al. [11], and $\mathrm{Hu}$ et al. [12]. Laser irradiation is widely applied for welding due to the non-contact nature of laser processing and high speed with low thermal load on the components, high aspect ratio of the joints, and deep penetration as reported by Von der Linde et al. [13]. Dahotre and Harimkar [14] reported a large number of factors involved in laser materials processing that need to be considered. For nanoparticle, size-dependent mechanical performance would be affected by various laser parameters including laser type, laser power beam dimensions, and laser wavelength as presented by $[5,9]$ and Khan et al. [15]. For instance, there is a method, which has been developed by Maity et al. [16], for selective thermal process of a collection of nanofibers by embedding gold nanoparticles within polymeric materials which are exposed to irradiation of violet diode laser at $405 \mathrm{~nm}$ and red $808 \mathrm{~nm}$ diode laser for photothermal heating. Photon energy transfer into localized heat affects the nanoparticle and its immediate vicinity. Having the ability to heat nanomaterials gives us the opportunity to manipulate the materials and change some characteristics while leaving the desired properties unaffected as mentioned by Garnett et al. [17].

At the most basic level, in the regime of laser nanomaterials processing conditions, a strong understanding of the interaction between laser irradiation and nanomaterials properties including material absorption and light intensity is crucial as studied by Cui [18]. Noble metal nanoparticles have strong plasmon resonance at the visible wavelengths of the electromagnetic spectrum. The surface plasmon resonance wavelength of the nanoparticles is affected by several parameters including particle size, shape, dielectric properties, and the refractive index of the surrounding matrix as reported by Khlebtsov et al. [19]. According to the studies conducted by Honda et al. [20], laser interaction with gold nanoparticle in liquid at laser frequencies close to the surface plasmon resonance efficiently generates heat, which is considered to be localized around nanoparticle. Liquid environment is an appropriate medium for laser assisted fabrication of nanomaterials and many of the laser applications for nanomaterials processing have been carried out in liquids [1-4,7,20]. Therefore, interaction of laser beam with nanoparticles in liquid is an important problem from both theoretical and experimental points of view. In this study, the interaction between nanosecond laser pulses and spherical gold nanoparticles dispersed in water is investigated for gold nanoparticle radius in the range of $2-40 \mathrm{~nm}$ at the laser wavelengths of $355 \mathrm{~nm}, 532 \mathrm{~nm}, 633 \mathrm{~nm}$, and $900 \mathrm{~nm}$ employing Mie theory and energy conservation during nanosecond laser pulse. Heating rate and maximum temperature of gold nanoparticles have been estimated via calculating the absorption efficiency and absorbed energy. As a staple topic of this research, the influence of laser wavelength and nanoparticle size on the heating process is also explored. In addition, size-dependent optical and thermal properties of gold nanoparticles are also taken into account in computations. The results are useful for a proper selection of laser parameters, specifically laser wavelength, for nanoparticles processing, photothermal phenomena, and other applications based on lasernanoparticle interaction in liquid.

\section{Methods and theory}

Nanoparticle heating and melting can be described in three steps including energy absorption from laser pulse, nanomaterials melting, and melt solidification. Schematic sketch of pulsed laser heating and surface melting of gold nanoparticles is depicted in Figure 1. Previous studies have shown the optical response of metal nanoparticles to be closely related with the localized surface plasmon resonance; therefore, energy transfer is determined by the absorption and scattering cross-section of nanoparticles that are essentially governed by excitation of surface plasmon resonance [19]. Mie theory estimates the optical cross-sections, including absorption and scattering cross-sections, of spherical metal nanoparticles. Interaction between an electromagnetic radiation and a homogenous particle and analytical calculation of Mie total extinction, scattering, and absorption cross-sections were discussed in detail by Bohren and Huffman [21].

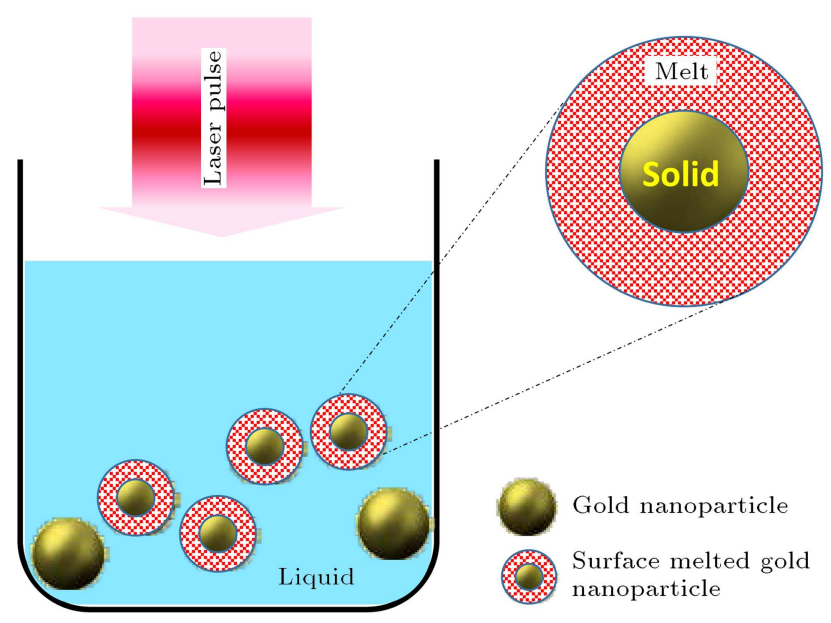

Figure 1. Schematic sketch of pulsed laser heating and surface melting of gold nanoparticles in liquid. 
For metal nanoparticle with a radius $r_{o}$ embedded in a dielectric matrix with the permittivity $\varepsilon_{m}$ when $r_{o}$ is much smaller than the laser wavelength $\lambda$, optical properties are determined by the absorption efficiency $K_{\text {abs }}\left(r_{o}, \lambda\right)$ that is obtained by Blaber et al. [22]:

$K_{\mathrm{abs}}=\frac{24 \pi r_{o} \varepsilon_{m}^{3 / 2}}{\lambda} \frac{\varepsilon^{\prime \prime}}{\varepsilon^{\prime \prime 2}+\left(\varepsilon^{\prime}+2 \varepsilon_{m}+\left(48 \pi^{2} r_{o}^{2} \varepsilon_{m}^{2} / 5 \lambda^{2}\right)\right)^{2}}$

where $\varepsilon=\varepsilon^{\prime}+i \varepsilon^{\prime \prime}$ is the dielectric function of the gold nanoparticle with the real $\varepsilon^{\prime}$ and imaginary $\varepsilon^{\prime \prime}$ parts. If $\varepsilon^{\prime}+2 \varepsilon_{m}+\left(48 \pi^{2} r_{o}^{2} \varepsilon_{m}^{2} / 5 \lambda^{2}\right)=0$, then the absorption is enhanced close to the surface plasmon resonance frequency due to a collective electron oscillation within gold nanoparticle. Deviations of the dielectric function of nanoparticles from the bulk dielectric function with the decreasing particle size to the value below the mean free pass of electrons is discussed by Ma et al. [23]. The size-dependent modified dielectric function is obtained as [23]:

$$
\varepsilon_{\text {size }}(\omega)=\varepsilon_{b-e}(\omega)+\left(1-\frac{\omega_{p}^{2}}{\omega^{2}+i\left(\Gamma_{\mathrm{bulk}}+\Gamma(r)\right) \omega}\right),
$$

where $\varepsilon_{b-e}$ is the interband transition term and the other part denotes the Drude term which considers conduction electrons. In addition, $\omega_{p}$ represents the frequency of volume plasma oscillations. $\Gamma_{\text {bulk }}$ is bulk volume decay constant and $\Gamma(r)$ is size-dependent volume decay parameter related to the electron mean free path $l_{\text {eff }}$ and Fermi velocity $v_{F}$ as $\Gamma(r)=v_{F} l_{\text {eff }}^{-1}$ [23].

An estimation of photothermal heating of gold nanoparticle under laser pulse requires the calculation of the total amount of absorbed laser pulse energy by gold nanoparticle. The energy of photons absorbed by gold nanoparticle $Q_{\text {abs }}$ and the thermal energy of the nanoparticle $E_{T}$ are given by Pustovalov et al. [24] as:

$$
\begin{aligned}
& Q_{\mathrm{abs}}(t)=\pi r_{o} \int_{0}^{t} I(t) K_{\mathrm{abs}} d t, \\
& E_{T}=\frac{4}{3} \pi r_{o}^{3} \rho_{o} c_{o} T,
\end{aligned}
$$

where $I, \rho_{o}, c_{o}$, and $T$ respectively stand for the laser intensity, the density of gold nanoparticle, the specific heat capacity of the gold nanoparticle, and the particle temperature. With the use of short laser pulse, of which the duration is much less than the thermal relaxation time, the particle has the ability to rapidly raise its temperature without substantially affecting the surroundings. The energy conservation law for nanoparticle can be given as [24]:

$$
Q_{\mathrm{abs}}-Q_{m}=E_{T}-E_{T_{\infty}},
$$

where $E_{T_{\infty}}$ is the initial value of the particle's thermal energy in liquid environment. In addition, $Q_{m}=$ $\rho_{o} V_{m} L_{m}$ represents the energy spent for partial melting of gold nanoparticle, $V_{m}$ is melt volume, and $L_{m}$ is the specific latent heat of fusion. In this calculation, $c$ and $L_{m}$ are assumed to be size-independent.

The dependency of the melting temperature of gold nanoparticle, $T_{m p}$, upon the size of the particles, considered for free-standing particles, is taken into account by Qi and Wang [25] and Nanda [26] as:

$$
T_{m p}=T_{m b}(1-d / 2 r)
$$

where $T_{m b}$ is melting temperature of bulk gold, $r$ stands for gold nanoparticle radius, and $d$ is the size of gold atom. As the size of nanospheres reduces, the melting temperature drastically decreases and must be considered in a photothermal heating process. In this research, all of the calculations were carried out using size-dependent parameters including dielectric function, absorption coefficient, and melting temperature of nanoparticles. The size-dependent melting point of gold nanospheres is calculated based on Eq. (6). All calculations were performed using MATLAB and the initial temperature of gold nanoparticles was $300 \mathrm{k}$. At first, for estimation of transferred laser energy to nanoparticles, the optical and plasmonic properties of gold nanoparticles with size of 2-40 $\mathrm{nm}$ have been calculated. For calculation of absorption efficiency of gold nanoparticle based on Eq. (1), the dielectric function of gold was calculated as a function of wavelength by linear interpolation of the values measured by Johnson and Christy [27]. Gold nanoparticles are irradiated to melt by using short laser pulses having a duration of $30 \mathrm{~ns}$ at several laser wavelengths including $355 \mathrm{~nm}$, $532 \mathrm{~nm}, 633 \mathrm{~nm}$, and $900 \mathrm{~nm}$ in ultraviolet, visible, and infrared regions, respectively. By employing short laser pulses, heat dissipation from pulsed laser heated gold nanoparticles to their vicinity can be ignored.

\section{Results and discussion}

To begin the investigation of the interaction between laser and gold nanoparticles, the efficiency of absorption is calculated on the basis of Eq. (1). Figure 2 represents absorption efficiency of gold nanoparticle as a function of the particle size and laser wavelength. As obviously seen from Figure 2(a), the absorption efficiency is very high when the radius of nanoparticles is between 42 to $50 \mathrm{~nm}$ at the wavelength of $532 \mathrm{~nm}$. At 355 and $532 \mathrm{~nm}$, the efficiency of absorption increases as the nanosphere radius is increased to 36 and $46 \mathrm{~nm}$, respectively. In addition, the maximum of optical absorption efficiency is situated at around $K_{\text {abs }}=$ 1.7 and $K_{\text {abs }}=5.5$ for laser irradiation at $355 \mathrm{~nm}$ and $532 \mathrm{~nm}$, respectively. No substantial change is shown for small nanoparticles absorption at the $633 \mathrm{~nm}$ 


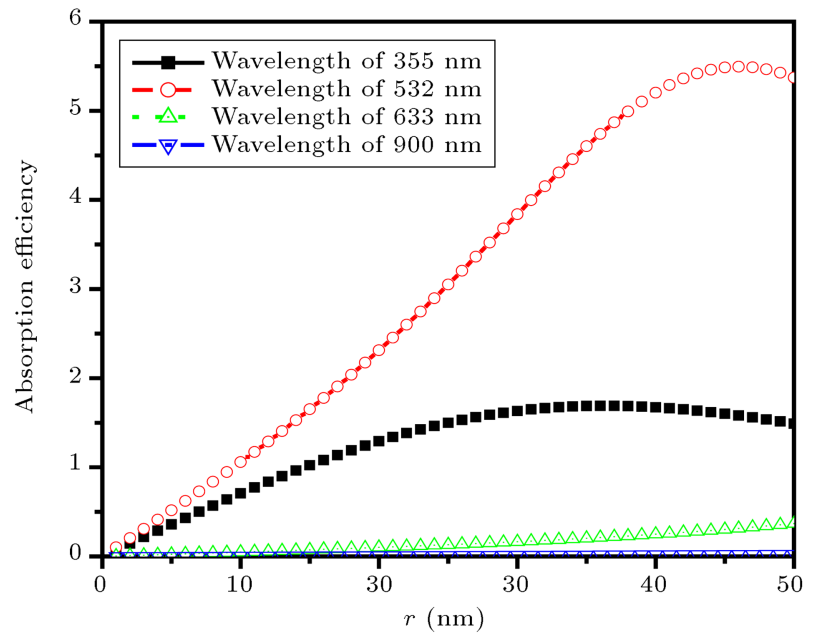

(a)

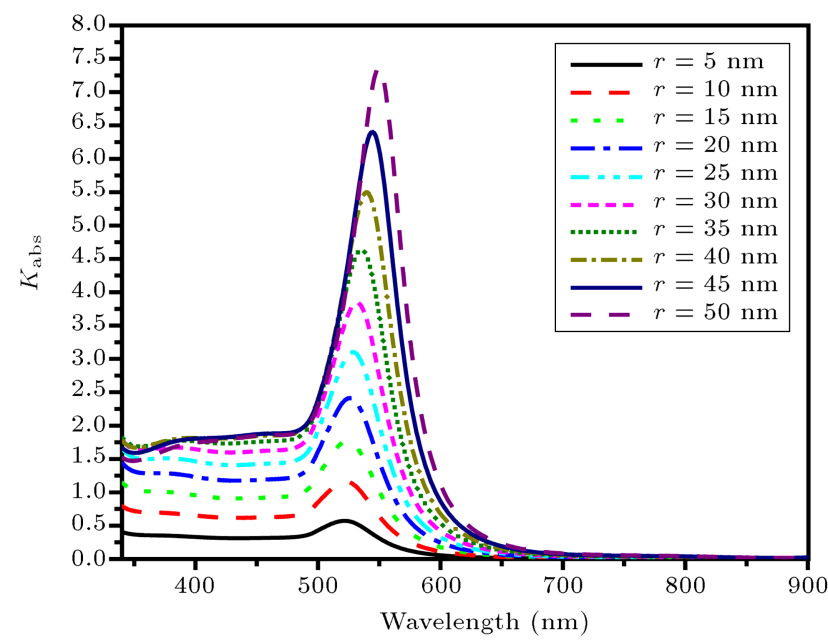

(b)

Figure 2. (a) Absorption efficiency factor versus the radius of the spherical gold nanoparticle dispersed in water. (b) Absorption efficiency at different laser wavelengths.

laser wavelength. There is almost no considerable change in the absorption efficiency by varying the dimensions in the near infrared range. As illustrated in Figure 2(b), maximum optical absorption is located at the wavelengths from around 521 to $549 \mathrm{~nm}$ for gold nanoparticles with radii between 5 to $50 \mathrm{~nm}$, respectively. The results demonstrate that large particles have a significant absorption at longer wavelengths. In addition, for the gold nanoparticle with radius of $40 \mathrm{~nm}$, the peak of the absorption is observed in the middle visible region around the wavelength of $540 \mathrm{~nm}$. Surface plasmon resonance is an effective mechanism of photon absorption by gold nanoparticles, especially at laser wavelength of $532 \mathrm{~nm}$. However, absorptions by free electrons and interband transition are more important at long and short laser wavelengths, respectively [21]. Laser exposure at plasmon resonance frequency results in an efficient photothermal heating of nanoparticles although photothermal heating by irradiation at wavelength far from the plasmon resonance is a more controllable process. Surface melting and forming a molten material is the main problem that especially affects the rate of temperature rising of the nanoparticle. As an application of these results, for instance, nanoscale welding and soldering can be obtained by controlling the optical energy, heating and melting process via adjusting welding filler size, and laser wavelength.

Figure 3 demonstrates the effects of nanoparticle size on photothermal heating and melting of gold nanoparticles at different laser wavelengths. The size of nanoparticle affects both gold nanoparticle melting and energy transfer rate. The rate of energy transfer to gold nanoparticle strongly depends on size-dependent absorption cross-section of the particle. Absorption cross-section is also related to the geometrical crosssection and size-dependent dielectric function of gold nanoparticle. Therefore, size effects are more important for ultrafine gold nanoparticles, especially for particles smaller than $10 \mathrm{~nm}$ as discussed by Scaffardi et al. [28].

As demonstrated in Figure 3(a), for 355 nm laser wavelength, the melting process of large gold nanoparticles begins with retardation; it takes a longer time to complete melting and at the end of the laser pulse, their maximum temperature reaches lower values than the temperature of small nanoparticles. For instance, temperature of large nanoparticle is about $1000 \mathrm{k}$ lower than the temperature of ultra-small nanoparticles. In addition, the temperature of ultra-small $4 \mathrm{~nm}$ gold nanoparticle rapidly rises up to around $2725 \mathrm{k}$. As can be seen in Figure 3(b), at $532 \mathrm{~nm}$ laser wavelength, gold nanoparticle with radius of $40 \mathrm{~nm}$ absorbs the laser pulse energy, heats, and melts faster than the $5 \mathrm{~nm}$ particle during the laser exposure. The origin of this behavior is the optical properties, especially surface plasmon resonance of radius $40 \mathrm{~nm}$ gold nanoparticles that is located at wavelength close to $532 \mathrm{~nm}$. Therefore, the frequency matching between laser and nanoparticle surface plasmon is very vital for obtaining an efficient photothermal heating of large nanoparticles. Heating of large particles is an important issue in size reduction of nanoparticles by laser irradiation or nanoparticle preparation via laser fragmentation of microparticles as studied by Lee et al. [29]. The maximum temperature is from around $1876 \mathrm{k}$ up to $2466 \mathrm{k}$ for nanoparticles with radius from $2 \mathrm{~nm}$ up to $40 \mathrm{~nm}$. Figure 3(c) demonstrates that ultra-small gold nanoparticles warm up slowly to about $1750 \mathrm{k}$ at the end of laser pulse in comparison with the large nanoparticles. In addition, the results demonstrate that the temperature of large gold nanoparticles could be raised to above $2300 \mathrm{k}$ under exposure of $633 \mathrm{~nm}$ laser pulse. As obviously seen in Figure 2(a), this 


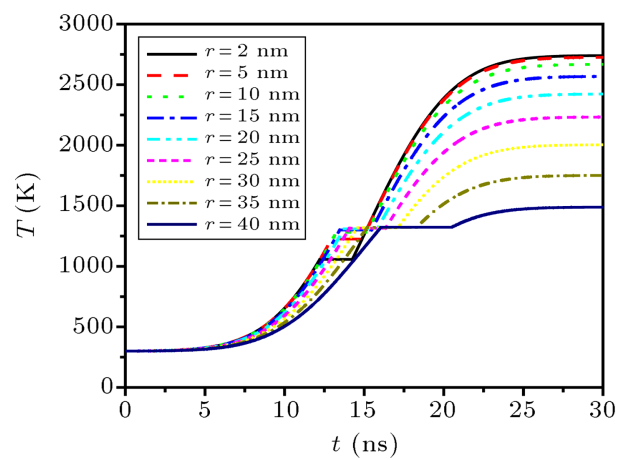

(a)

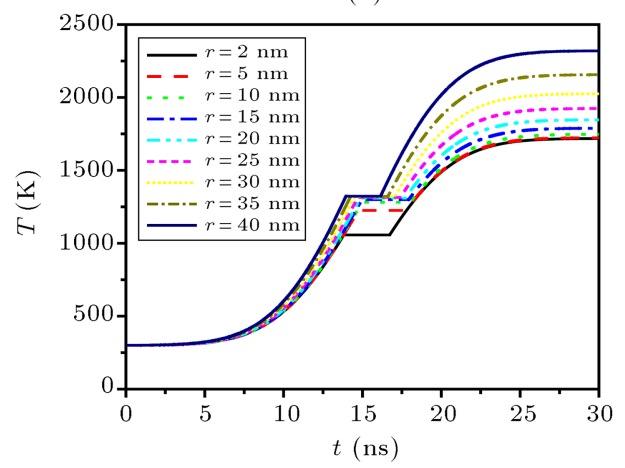

(c)

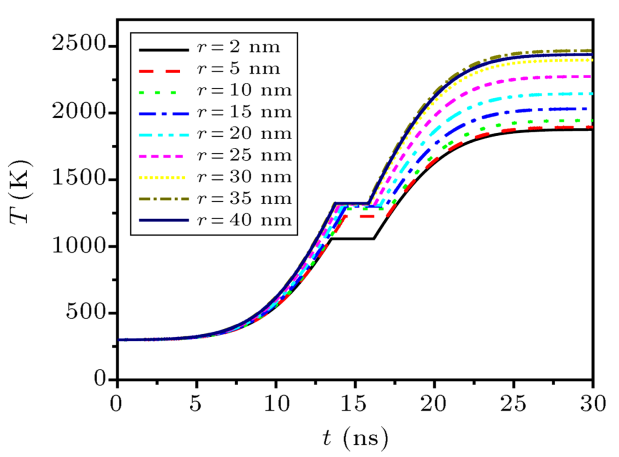

(b)

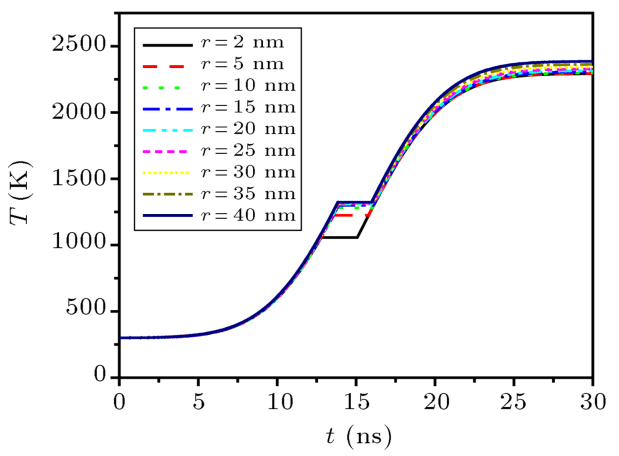

(d)

Figure 3. Temperature versus time at the laser wavelengths of (a) $355 \mathrm{~nm}$, (b) $532 \mathrm{~nm}$, (c) $633 \mathrm{~nm}$, and (d) $900 \mathrm{~nm}$ with respect to the dependency of melting point of gold nanoparticles upon the particle size.

behavior is consistent with the size-dependent optical properties of gold nanoparticles at $630 \mathrm{~nm}$. For laser irradiation at $900 \mathrm{~nm}$, as clearly shown in Figure 3(d), the temperature of gold nanoparticles reaches about $2290 \mathrm{k}$ up to $2390 \mathrm{k}$ at the end of the laser pulse. The rises in temperature for all gold nanoparticles up to around $1000 \mathrm{k}$ are nearly the same at the laser wavelength of $900 \mathrm{~nm}$ due to the weak absorption of gold nanoparticle at this wavelength. Therefore, long wavelength lasers are appropriate preferences for homogeneous heating of a collection of nanoparticles with broad size distribution.

As obviously seen in Figure 3, the maximum temperatures and photothermal heating rates of gold nanoparticles with radii of $2 \mathrm{~nm}$ and $40 \mathrm{~nm}$ could be compared in the near ultraviolet, visible, and near infrared ranges. As illustrated in Figure 3(a), for $4 \mathrm{~nm}$ gold nanoparticle, temperature rising is fast at $355 \mathrm{~nm}$. In addition, complete melting needs more time in case of longer laser wavelengths. Here, being able to generate extreme localized heat leads to efficient and significant rise in temperature and quick heating process. A rapid heating can result in improved properties without thermal damage or ambient environmental degradation [17].

In Figure 4, the beginning times of the melting and in Figure 5, the times that gold nanoparticle melting was completely accomplished have been compared for different sizes of gold nanoparticles at the laser

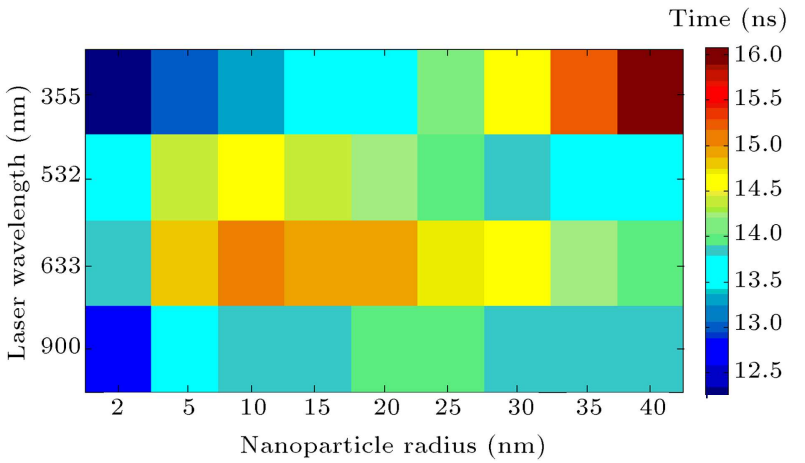

Figure 4. The beginning time of the size-dependent melting process for different sizes of gold nanoparticles at laser wavelengths in ultraviolet, visible, and near infrared regions.

wavelengths of $355,532,633$, and $900 \mathrm{~nm}$. At laser wavelength of $355 \mathrm{~nm}$, the rises in temperatures of small-size nanoparticles and laser induced melting are quick; however, larger nanoparticles warm slower. At this wavelength, larger nanoparticles are more suitable for large area heating, for instance large area soldering, and small nanoparticles are more useful for localized photothermal heating processes.

At laser wavelength of $900 \mathrm{~nm}$, temperature of gold nanoparticle rises in the same way for all sizes of nanoparticles. Melting with this wavelength is useful for heating in a process with broad-size distribution gold nanoparticle; however, some thermal effects may 


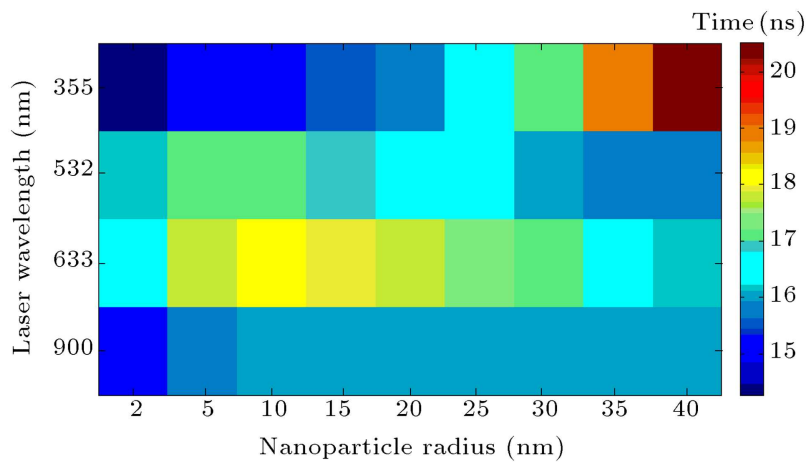

Figure 5. The times that gold nanoparticle melting was completely accomplished for different sizes of nanoparticles at laser wavelengths in ultraviolet, visible, and near infrared regions.

possibly be observed. The calculations show that the temperature in a heating process in presence of gold nanoparticles can rise only via adjusting the nanoparticle size without increasing the laser pulse energy. The results provide a way to select appropriate laser wavelength and optimized nanoparticle size for smalland large-area heating below materials or environment damage threshold energy. The calculation indicates that nanosecond lasers can provide an appropriate melting of nanoparticles if the incident photons energy is properly adjusted.

According to Figures 4 and 5, the results for $532 \mathrm{~nm}$ laser wavelength demonstrate that the melting is clearly most efficient when the laser illumination frequency occurs at the localized surface plasmon resonance frequency of gold nanoparticles. In addition, dependency of heating rate and melting process on the size of nanoparticles is stronger at laser frequencies close to surface plasmon resonance frequency of nanoparticles, which in this case is $532 \mathrm{~nm}$.

These findings can also contribute to the clarification of effects and applications of the pulsed laser process, which locally concentrates the laser power densities with minimum heat inputs, forming very small and localized affected regions while avoiding excessive damage. The calculations are useful in nanoparticle-enhanced surface engineering and localized laser heating, and surface deformation and particles fragmentation by laser irradiation with only simple control of laser parameters and nanoparticle size. In addition, the ability to adjust energy deposition in the heating area is an advantage of using laser beam as the energy source in heating processes, especially in localized heating cases in attendance of plasmonic nanoparticles as discussed by Guo [30].

\section{Conclusions}

In this research, nanosecond pulsed laser heating and melting of dispersed gold nanoparticles in water at different laser wavelengths are investigated. The results display that both the laser wavelength and the particle size have a profound effect on the laser heating and melting of gold nanoparticles. The calculated results demonstrate that laser heating with $532 \mathrm{~nm}$ wavelength is more effective due to the surface plasmon resonance of gold nanoparticles, especially for heating and melting at low-intensity laser beam. Short laser wavelength at $355 \mathrm{~nm}$ is applicable for selective heating of small nanoparticles. At long wavelengths, heating of nanoparticles is size-independent, which is applicable for heating colloidal solution and broad size distribution, simultaneously. The results provide a way for estimation of optimized laser wavelength and nanoparticle size for a laser heating process at a specific temperature by minimum laser irradiation at the lowest level of damage on materials.

\section{References}

1. Kadhim, R.G., Ali, A.K. and Noori, M.F. "Nanofabrication and characterization of gold metal by pulsed laser ablation in SDS solution", Advances in Physics Theories and Applications, 11, pp. 23-27 (2012).

2. Mafune, F., Kohno, J., Takeda, Y. and Kondow, T. "Formation of gold nanonetworks and small gold nanoparticles by irradiation of intense pulsed laser onto gold nanoparticles", Journal of Physical Chemistry B, 107, pp. 12589-12596 (2003).

3. Muniz-Mirande, M., Gellini, C., Simonelli, A., Tiberi, M., Giammanco, F. and Giorgetti, E. "Characterization of copper nanoparticles obtained by laser ablation in liquids", Applied Physics A: Materials Science \& Processing, 110(4), pp. 829-833 (2013).

4. Warner, D., Hashimoto, S. and Uwada, T. "Remarkable photothermal effect of interband excitation on nanosecond laser-induced reshaping and size reduction of pseudo-spherical gold nanoparticles in aqueous solution", Langmuir, 26, pp. 9956-9963 (2010).

5. Son, M., Kim, S.J., Kim, J.Y. and Jang, D.J. "Laserinduced silver nanojoining of gold nanoparticles", Journal of Nanoscience and Nanotechnology, 13(8), pp. 5777-5782 (2013).

6. Mafune, F., Kohno, J., Takeda, Y. and Kondow, T. "Nanoscale soldering of metal nanoparticles for construction of higher-order structures", Journal of the American Chemical Society, 125, pp. 1686-1687 (2003).

7. Cui, Q., Gao, F., Mukherjee, S. and Gu, Z. "Joining and interconnect formation of nanwires and carbon nantubes for nanoelectronics and nanosystems", Small, 5, pp. 1246-1257 (2009).

8. Guo, J.Y., Xu, C.X., Hu, A.M., Shi, Z.L., Sheng, F.Y., Dai, J. and Li, Z.H. "Welding of gold nanowires with different joining procedures", Journal of Nanoparticle Research, 14(666), pp. 1-12 (2012). 
9. Hu, A., Panda, S.K., Khan, M.I. and Zhou, Y. "Laser welding, microwelding, nanowelding and nanoprocessing", Chinese Journal of Lasers, 36(12), pp. 3149-3159 (2009).

10. Kim, S.J. and Jang, D. J. "Laser-induced nanowelding of gold nanoparticles", Applied Physics Letters, 86(3), pp. 331121-331123 (2005).

11. Zhou, Y., Hu, A., Khan, M.I., Wu, W., Tam, B. and Yavuz, M. "Recent progress in micro and nano-joining", Journal of Physics: Conference Series, 165(1), pp. 1-6 (2009).

12. Hu, A., Zhou, Y. and Duley, W.W. "Femtosecond laser-induced nanowelding: fundamentals and applications", The Open Surface Science Journal, 3, pp. 42-49 (2011).

13. Von der Linde, D., Sokolowski-Tinten, K. and Biakowski, J. "Laser-solid interaction in the femtosecond time regime", Applied Surface Science, 109-110, pp. 1-10 (1997).

14. Dahotre, N.B. and Harimkar, S.P., Laser Fabrication and Machining of Materials, Springer, New York, pp. 412-447 (2008).

15. Khan, M.I., Panda, S.K. and Zhou, Y. "Effects of welding parameters on the mechanical performance of laser welded nitinol", Materials Transactions, 49(11), pp. 2702-2708 (2008).

16. Maity, S., Kozek, K.A., Wu, W., Tracy, J.B., Bochinski, J.R. and Clarke, L.I. "Anisotropic thermal processing of polymer nanocomposites via the photothermal effect of gold nanorods", Particle and Particle Systems Characterization, 30, pp. 193-202 (2013).

17. Garnett, E.C., Cai, W., Cha, J.J., Mahmood, F., Connor, S.T., Christoforo, M.G., Cui, Y., McGehee, M.D. and Brongersma, M.L. "Self-limited plasmonic welding of silver nanowire junctions", Nature Materials, 11, pp. 241-249 (2012).

18. Cui, B., Recent Advances in Nanofabrication Techniques and Applications, pp. 113-140, InTech (2011).

19. Khlebtsov, N.G., Trachuk, L.A. and Mel'nikov, A.G. "The effect of the size, shape, and structure of metal nanoparticles on the dependence of their optical properties on the refractive index of a disperse medium", Optics and Spectroscopy, 98, pp. 77-83 (2005).

20. Honda, M., Saito, Y., Smith, N.I., Fujita, K. and Kawata, S. "Nanoscale heating of laser irradiated single gold nanoparticles in liquid", Optics Express, 19(13), pp. 12375-12383 (2011).

21. Bohren, C. and Huffman, D., Absorption and Scattering of Light by Small Particles, A Wiley-International Publication, New York (1983).

22. Blaber, M.G., Arnold, M.D., Harris, N., Ford, M.J. and Cortie, M.B. "Plasmon absorption in nanospheres: a comparison of sodium, potassium, aluminum, silver, and gold", Physica B, 394, pp. 184-187 (2007).

23. Ma, X., Lu, J.Q., Brock, R.S., Jacobs, K.M., Yang, P. and Hu, X.H. "Determination of complex refractive index of polystyrene microspheres from 370 to 1610 nm", Physics in Medicine and Biology, 48, pp. 41654172 (2003).

24. Pustovalov, V.K. and Babenko, V.A. "Optical properties of gold nanoparticles at laser radiation wavelengths for laser applications in nanotechnology and medicine", Laser Physics Letters, 1(10), pp. 516-520 (2004).

25. Qi, W.H. and Wang, M.P. "Size effect on the cohesive energy of nanoparticle", Journal of Materials Science Letters, 21(22), pp. 1743-1745 (2002).

26. Nanda, K.K. "Size-dependent melting of nanoparticles: hundred years of thermodynamic model", Pramana Journal of Physics, 72(4), pp. 617-628 (2009).

27. Johnson, P.B. and Christy, R.W., "Optical constants of the noble metals", Phys. Rev. B, 6, pp. 4370-4379 (1972).

28. Scaffardi, L.B., Pellegri, N., de Sanctis, O. and Tocho, L.O. "Sizing gold nanoparticles by optical extinction spectroscopy", Nanotechnology, 16, pp. 158-163 (2005).

29. Lee, J., Becker, M.F. and Keto J.W. "Dynamics of laser ablation of microparticles prior to nanoparticle generation", J. Appl. Phys., 89, pp. 8146-8152 (2001).

30. Guo, K.W. "A review of micro/nano welding and its future developments", Recent Patents on Nanotechnology, 3, pp. 53-60 (2009).

\section{Biographies}

Samaneh Malek has a BSc degree in Physics from Kharazmi University, Iran, and an MSc degree in Physics from Shahed University, Tehran, Iran. Her MSc degree thesis was entitled: "study of the laser heating of gold nanoparticles in cell targeting". Her research areas include laser matter interaction, optical properties of metallic nanomaterials, and laser heating and welding of nanoparticles.

Reza Poursalehi has $\mathrm{PhD}, \mathrm{MSc}$, and BSc degrees in Physics from Sharif University of Technology, Tehran, Iran. He joined the Nanomaterials Group in the Department of Materials Engineering, Tarbiat Modares University, in 2012. His interests include laser ablation in liquid, pulsed laser deposition, laser nanomaterials processing, optical properties of nanomaterials, and smart nanocomposites thin film membranes. 